\title{
The Born Formula Describes Enthalpy of Ions Solvation
}

\author{
Nikolai Bazhin \\ Institute of Chemical Kinetics and Combustion, Novosibirsk State University, Novosibirsk 630090, Russia \\ Correspondence should be addressed to Nikolai Bazhin, bazhin8999@kinetics.nsc.ru
}

Received 3 February 2012; Accepted 26 February 2012

Academic Editor: M. Manciu

Copyright (C) 2012 Nikolai Bazhin. This is an open access article distributed under the Creative Commons Attribution License, which permits unrestricted use, distribution, and reproduction in any medium, provided the original work is properly cited.

The process of ion solvation has been studied in the reversible system using the Van't Hoff equilibrium box. It is shown that the Born formula for solvation energy describes a change in enthalpy rather than in Gibbs energy.

\section{Introduction}

Solvation energy is usually taken as the difference in work which results from ion charging in both vacuum and liquid phase due to a mental process [1]. For an approximate, simple estimate of solvation energy, one can use the Born formula [1] according to which the solvation energy $\left(E_{\text {solv }}\right)$ is the difference in the energy of charging the sphere of radius $a$ to charge $Z e$ in both vacuum and medium with a dielectric constant $\mathcal{E}$

$$
E_{\text {solv }}=\frac{Z^{2} e^{2}}{8 \pi \varepsilon_{0} a}\left(1-\frac{1}{\varepsilon}\right) N_{\mathrm{A}},
$$

where $\varepsilon_{0}$ is an electric constant, and $N_{\mathrm{A}}$ is the Avogadro number. According to Born, (1) is the work obtained by transferring a charged sphere from vacuum to condensed medium. The work manifests itself in solvation heat [1]. Since the system temperature is constant, the heat emitted passes to a thermostat. It is assumed then that Born has taken solvation energy as the change in either internal energy or enthalpy upon solvation. However, discussing Born's work, the authors [2] assert that without any doubts, value (1) calculated by Born is the maximum work performed by electric forces during ion dissolving. Therefore, in [2], (1) is equated with the change in the Gibbs energy which allowed the authors [2] to calculate solvation enthalpy as

$$
\Delta H_{\text {solv }}=\frac{Z^{2} e^{2}}{8 \pi \varepsilon_{0} a}\left(1-\frac{1}{\varepsilon}-\frac{T}{\varepsilon^{2}} \cdot \frac{d \varepsilon}{d T}\right) N_{\mathrm{A}} .
$$

Equation (1) fails to quantitatively describe ion solvation in numerical form. The advantage is that it can be used to qualitatively estimate the dependence of solvation energy on ion size, medium's dielectric constant, and temperature. It is of importance then to ascertain whether (1) is the Gibbs function or the solvation enthalpy.

We will omit here the problems of the applicability of (1), considered, for example, in [3-10].

Since the energy of charging is the work, formula (1) is commonly accepted as the change in the Gibbs function upon solvation which causes, however, some doubts for the following reasons.

(1) To calculate the change in the Gibbs function, one should accurately determine the initial and final states of the system. To derive formula (1), the charging of only one particle is considered, and the result obtained is multiplied by the Avogadro number to calculate the charging energy per one mole. As compared with enthalpy, in the initial state, the Gibbs function is pressure dependent, and, in the final state, it depends on ion concentration. Thus, it is impossible to describe the change in the Gibbs function in terms of (1).

(2) There is no evidence that formula (1) is sure to describe the change in the Gibbs function rather than the change in other values, for example, in enthalpy.

The goal of the present work is to solve this problem by means of the Van't Hoff equilibrium box (VHEB) proposed by Van't Hoff to derive an equilibrium constant. To this end, the manuals on chemical thermodynamics $[11,12]$ consider the VHEB as an old alternative of the method of chemical potentials. At present, the VHEB is very useful to 
solve qualitative problems. Thus, for example, in [13], the VHEB was used to demonstrate that the reversible work at $T, p=$ Const in the systems, including chemical reactions, arises only due to the thermal energy of the environment, and the energy evolved by reactions is not employed to produce useful work.

\section{Solvation}

The change in the Gibbs function between two states of the system is independent of the type of transition between these states. This transition can be either reversible or irreversible. However, to calculate the change in the Gibbs function, the transition should be reversible because, in this case, the calculation of the change in the Gibbs function becomes more available. The reversible solvation process is performed in this paper by analogy as described in [13] for coal oxidation. The system under study is considered ideal.

Let us describe first the standard initial and the final states. The final state is the electrolyte solution. Due to Alberty [14], the standard state of an electrolyte solution is "the hypothetically ideal solution at unit activity in which ions have the properties they do at infinite dilution." We assume then that a standard state of ions in gas is a hypothetical gas at 1 bar, in which the ions have the properties they do at infinite expansion. Thus, in these hypothetical standard states, the electrostatic interaction of ions is cut off which alleviates the calculation of thermodynamic values, describing the process of solvation.

Consider now the solvation process. Gaseous ions are assumed to occupy a very large volume. In addition, the volume of the liquid-phase system is also assumed very large. The system is located in a thermostat. Let us reversibly convert the part of the gaseous system from the initial state into the final one. We calculate the change in the thermodynamic functions upon transition of a hypothetical system from the standard state in the gas phase to that in the liquid state in the course of a conceptual process, consisting of two stages.

Step 1. At step 1 we take 1 mole of ions in the gaseous state at $p_{\text {st }}=1 \mathrm{bar}$ and expand the ion gas reversible to pressure $p_{\text {fin }}$, at which the chemical potential of ions in gas becomes equal to that in solution. The reversible process is accompanied obligatory by useful work production ( $\left.w_{\text {useful }}\right)$, and the change in the Gibbs function $\left(\Delta G_{1}\right)$ of the ions is equal to $w_{\text {useful }}$

$$
\Delta G_{1}=w_{\text {useful }}=R T \ln \left(\frac{p_{\text {fin }}}{p_{\text {st }}}\right) .
$$

The change in enthalpy $\left(\Delta H_{1}\right)$ of ideal ion gas expansion is of the form

$$
\Delta H_{1}=0 .
$$

The value of $p_{\text {fin }}$ is unknown. Value (3) cannot be given in terms of system parameters in the framework of the approach used. Upon expansion, the reversible work is performed which can be converted into the potential energy of the load. The work is performed only by the thermal thermostat energy because, in the system, there are no other energy sources. Thus, the change in the thermostat enthalpy $\left(\Delta H_{1 \text { thrermostat }}\right)$ during gas expansion at the first step is

$$
\Delta H_{1 \text { thrermostat }}=R T \ln \left(\frac{p_{\mathrm{fin}}}{p_{\mathrm{st}}}\right) .
$$

Step 2. At the final pressure $p_{\text {fin }}$, we slowly introduce ions from the gas phase to solution through, for example, a semipermeable membrane. In this case, the Gibbs function does not change because of the equality of the chemical potentials of ions in the gas and liquid phases, and, thus,

$$
\Delta G_{2}=0 .
$$

Hence, the full change in the Gibbs function upon solvation process $\left(\Delta G_{\text {solv }}\right)$ is equal to useful work of the ion gas expansion during first step

$$
\Delta G_{\text {solv }}=\Delta G_{1}+\Delta G_{2}=R T \ln \left(\frac{p_{\text {fin }}}{p_{\text {st }}}\right) .
$$

It is worth noting that no relation was established between $\Delta G_{\text {solv }}$ and formula (1).

The process of solvation results in heat release. The reaction of ion dissolution is similar to that of coal oxidation [13]. The value of the change in the ions enthalpy at the second step $\left(\Delta H_{2}\right)$ is calculated from (1) because, in terms of the law of energy conservation, the difference in work on sphere charging in both vacuum and liquid should convert into another type of energy, represented in our case, by the solvation reaction heat

$$
\Delta H_{2}=-\frac{Z^{2} e^{2}}{8 \pi \varepsilon_{0} a}\left(1-\frac{1}{\varepsilon}\right) N_{\mathrm{A}} .
$$

Hence, the full enthalpy of solvation process $\left(\Delta H_{\text {solv }}\right)$ is calculated via the Born formula (1)

$$
\Delta H_{\text {solv }}=\Delta H_{1}+\Delta H_{2}=-\frac{Z^{2} e^{2}}{8 \pi \varepsilon_{0} a}\left(1-\frac{1}{\varepsilon}\right) N_{\mathrm{A}} .
$$

The change in the thermostat enthalpy at the second step $\left(\Delta H_{2 \text { thrermostat }}\right)$ during gas expansion is

$$
\Delta H_{2 \text { thrermostat }}=-\Delta H_{2} .
$$

The full thermostat enthalpy change in the reversible process is

$$
\begin{aligned}
\Delta H_{\text {thrermostat }} & =\Delta H_{1 \text { thrermostat }}+\Delta H_{2 \text { thrermostat }} \\
& =R T \ln \left(\frac{p_{\text {fin }}}{p_{\text {st }}}\right)+\frac{Z^{2} e^{2}}{8 \pi \varepsilon_{0} a}\left(1-\frac{1}{\varepsilon}\right) N_{\mathrm{A}} .
\end{aligned}
$$

In the case of the irreversible process $\left(w_{\text {useful }}=0\right)$, the full thermostat enthalpy change is equal to

$$
\begin{gathered}
\Delta H_{\text {thrermostat }}=\frac{Z^{2} e^{2}}{8 \pi \varepsilon_{0} a}\left(1-\frac{1}{\varepsilon}\right) N_{\mathrm{A}}, \\
\Delta H_{\text {thrermostat }}=-\Delta H_{\text {solv }} .
\end{gathered}
$$


The change in the Gibbs function (7) cannot be equated to the negative value of solvation energy (1) because the change in the Gibbs function in the reversible process determines the value of useful, reversible work rather than of the heat emitted.

Thus, the Born function (1) can be used to calculate only the solvation enthalpy.

\section{References}

[1] M. Born, "Volumen und Hydratationswarme der Ionen," Zeitschrift für Physik, vol. 1, pp. 45-48, 1920.

[2] N. Bjerrum and E. Larson, "Studien uber Ionenverteilungskoeffizienten I.," Zeitschrift für Physikalische Chemie, vol. 127, pp. 358-384, 1927.

[3] P. W. Atkins and A. J. MacDermott, "The born equation and ionic solvation," Journal of Chemical Education, vol. 59, no. 4, pp. 359-360, 1982.

[4] P. M. Qureshi, R. K. Varshney, and S. B. Singh, "Empirical correlations for the evaluation of the free energies of solvation of some gaseous monovalent ions," Journal of Chemical Education, vol. 66, no. 11, pp. 903-906, 1989.

[5] D. Bashford and D. A. Case, "Generalized born models of macromolecular solvation effects," Annual Review of Physical Chemistry, vol. 51, pp. 129-152, 2000.

[6] P. K. Yang and C. Lim, "The importance of excluded solvent volume effects in computing hydration free energies," Journal of Physical Chemistry B, vol. 112, no. 47, pp. 14863-14868, 2008.

[7] B. Roux, H. A. Yu, and M. Karplus, "Molecular basis for the born model of ion solvation," Journal of Physical Chemistry, vol. 94, no. 11, pp. 4683-4688, 1990.

[8] G. F. de Lima, H. A. Duarte, and J. R. Pliego, "Dynamical discrete/continuum linear response shells theory of solvation: convergence test for $\mathrm{NH}_{4}{ }^{+}$and $\mathrm{OH}^{-}$ions in water solution using DFT and DFTB methods," Journal of Physical Chemistry $B$, vol. 114, no. 48, pp. 15941-15947, 2010.

[9] C. S. Babu and C. Lim, "Theory of ionic hydration: insights from molecular dynamics simulations and experiment," Journal of Physical Chemistry B, vol. 103, no. 37, pp. 7958-7968, 1999.

[10] F. Hirata, P. Redfern, and R. M. Levy, "Viewing the born model for ion hydration through a microscope," International Journal of Quantum Chemistry, vol. 15, pp. 179-190, 1988.

[11] Ya. I. Gerasimov, Ed., Physical Chemistry, Chimiya, Moscow, Russia, 1980.

[12] L. E. Steiner, Introduction to Chemical Thermodynamic, McGraw-Hill, New York, NY, USA, 2nd edition, 1948.

[13] N. M. Bazhin and V. N. Parmon, "Conversion of the chemical reaction energy into useful work in the Van't Hoff equilibrium box," Journal of Chemical Education, vol. 84, no. 6, pp. 10531055, 2007.

[14] R. A. Alberty, Physical Chemislry, John Wiley \& Sons, New York, NY, USA, 7th edition, 1987. 

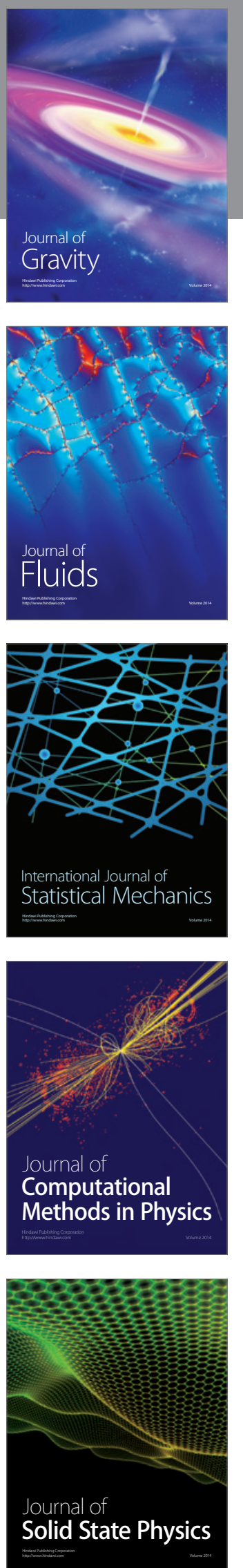
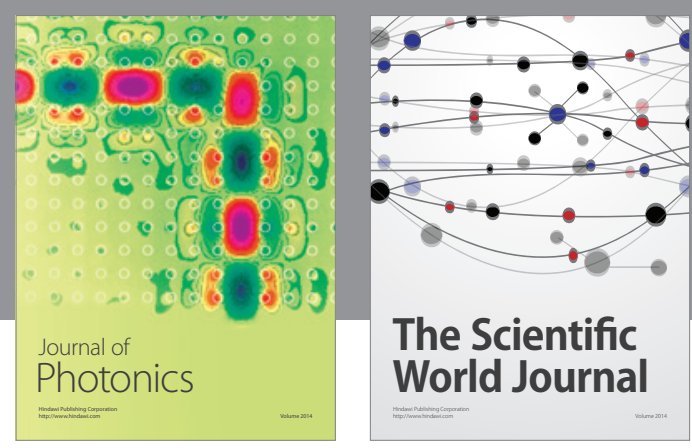

The Scientific World Journal

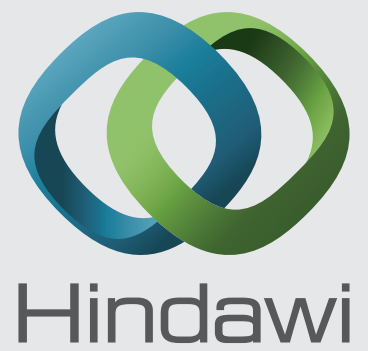

Submit your manuscripts at http://www.hindawi.com
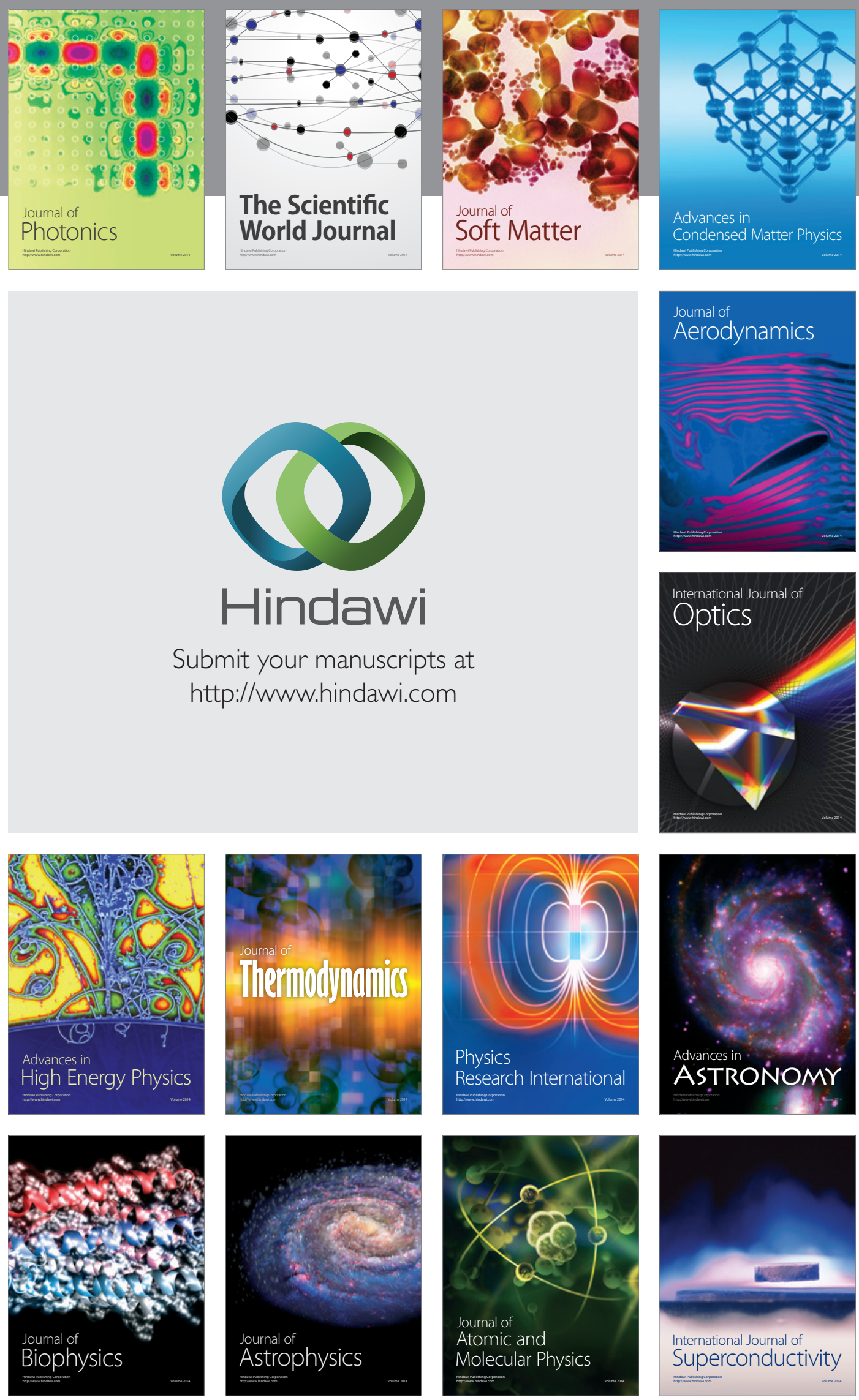
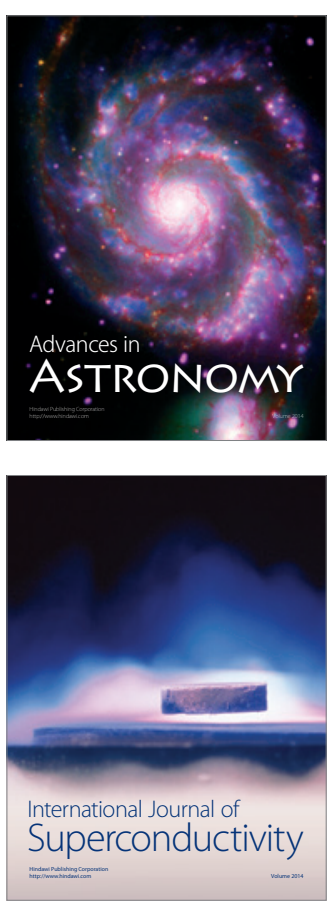